\title{
Fauna silvestre asociada a ganado vacuno doble propósito en sistema de silvopastoreo, Pinto, Magdalena, Colombia
}

\section{Association between wildlife and bovine dual purpose cattle in a silvopastured system, Pinto, Magdalena, Colombia}

\author{
Luz Botero A, ${ }^{1 *}$ M.Sc, Jaime De La Ossa V. ${ }^{1}$ Ph.D.
}

\begin{abstract}
${ }^{1}$ Universidad de Sucre. Facultad de Ciencias Agropecuarias. Grupo Biodiversidad Tropical. SincelejoColombia.*Correspondencia: Ibotero15@yahoo.es.
\end{abstract}

Recibido: Mayo de 2010; Aceptado: Julio de 2011.

\section{RESUMEN}

Objetivo. Evaluar la biodiversidad faunística asociada a ganadería vacuna de doble propósito en dos potreros estructurados con Botriochloa pertusa (colosoana) como herbácea dominante, uno de ellos plantado con Crescentia cujete (Bignoniaceae) como fuente de alimento adicional y otro con escaso y disperso arbolado. Materiales y métodos. El área de estudio está ubicada en el municipio de Pinto, Magdalena. Cada potrero evaluado fue de 10 ha, se mantuvo en cada uno un lote de quince vacas ( $1 / 2$ Cebú, $1 / 4$ Pardo y $1 / 4$ Costeño con Cuernos), la separación entre las áreas muestreadas fue de $500 \mathrm{~m}$. Los registros de fauna se condujeron mediante punto fijo de conteo, en diez sitios de avistamiento, durante 20 días continuos, con cubrimiento visual amplio. Al ganado vacuno adulto presente en las dos áreas de trabajo se le realizó un pesaje individual al inicio del trabajo y al final del mismo, con el fin de determinar posibles diferencias entre ellos. Resultados. Estadísticamente el número de individuos y el número de especies presentaron diferencias significativas, siendo mayor la diversidad biológica para el potrero que poseía la plantación de C. cujete, mientras que el peso corporal de las vacas sometidas a pastoreo en las dos áreas no presentó diferencias significativas. Conclusiones. Se demuestra la importancia de este modelo pecuario como promotor de la conservación faunística local al convertirse en refugio de variadas especies silvestres, al tiempo que mantiene la productividad existente sin alterar el ambiente sustancialmente.

Palabras clave: Biodiversidad, conservación, Crescentia cujete, fauna, ganado de doble propósito (Fuente: $C A B$ ). 


\section{ABSTRACT}

Objective. To evaluate the association between wild fauna biodiversity and dual purpose cattle in a silvopastured system, municipality of Pinto, Magdalena, Colombia. Animals were maintained in two different yards: yard 1) presented Botriochloa pertusa (colosoana) pastures as herbaceous dominant plus Crescentia cujete (Bignoniaceae) as source of additional food, yard 2) presented Botriochloa pertusa (colosoana) pastures as herbaceous dominant plus scarce and disperse trees. Materials and methods. A total of 15 cows ( $1 / 2$ zebu, brown $1 / 4$ and costeño con cuernos $1 / 4$ ) were located in a 10 ha, yard. The separation between the yards areas was $500 \mathrm{~m}$. The fauna was monitoring, in 10 fixed different places, with wide view, during 20 continuous days. Cows were weighted the first day and the last day of the experiment, with the purpose of determining possible weight differences among them. Results. The biological diversity of yard 1 (plantation of $C$. cujete), was different in number of individuals and number of species from yard 2 (scarce and disperses trees). However the corporal weight of the cows was not different between both yards.

Conclusions. This study demostrated the possibility to promote this kind of model for the refuge and conservation of a diversity of local fauna. without substantially altering the environment.

Key words: Biodiversity, conservation, Crescentia cujete, dual purpose cattle, fauna (Source: $C A B)$.

\section{INTRODUCCIÓN}

El aislamiento de las áreas remanentes de la tala, tiene importantes consecuencias para la biota y estas varían con el tiempo desde el momento del aislamiento, la distancia hasta los otros remanentes y el grado de conexión entre ellos. No obstante, no se ha profundizado en aspectos relacionados con la plantación de arbóreas y arbustivas en zonas previamente taladas para uso agropecuario, que actúan como reservorios importantes de la biota remanente y que llegan en cierta medida, dependiendo de sus características ecológicas a ser verdaderos refugios (1).

La complejidad de los sistemas forestales que involucran actividad antropogénica plantea la importancia de los efectos de los claros, bordes cercanos y diferentes formas de disturbio en bosques neotropicales, en los cuales es evidente que factores de regeneración natural o inducida ejercen efectos positivos o negativos sobre el componente biótico (2).

Es claro que existe un conflicto entre ganadería y conservación de la diversidad biológica, las causas son variadas pero están conectadas con la ocupación, transformación y uso del suelo y sus recursos, aunque exhiben particularidades las generalidades son innegables (3).

Como causas directas de la extinción de especies de vida silvestre en América Latina, así como en Colombia, se tienen: la deforestación, la transformación de hábitat y ecosistemas, la construcción de vías y otras obras de infraestructura, la introducción de especies exóticas, la sobreexplotación, la contaminación y el cambio climático (4). Cada uno de los impactos negativos antes señalados se relaciona con la ganadería; se puede examinar como ejemplo las causas de la deforestación en donde la colonización y expansión de la frontera agrícola y ganadera, así como la producción maderera, el consumo de leña, los incendios forestales y las plantaciones ilegales, son las causas de mayor impacto (5).

Aunque los agroecosistemas más complejos que puedan darse no soportan una mayor biodiversidad que la que pueda registrase en los ecosistemas naturales desplazados, existen algunos agroecosistemas que 
soportan la biota local como único refugio disponible y su diversidad adquiere importancia conservacionista especial; la ganadería puede reemplazar sistemas de alta productividad biológica y tornarlos en ambientes pobres, pero también puede ayudar a conservar una porción de la fauna y flora nativa (3). Como evidencia de ambas situaciones, se tiene que en las zonas de colonización de Colombia, mayoritariamente los bosque nativos se han transformado en pastizales homogéneos (6); pero también se puede señalar a manera de ejemplo, que en medio de extensos monocultivos de caña de azúcar en el Valle del Cauca, pequeñas áreas con sistemas silvopastoriles soportan una avifauna más rica que la de áreas circundantes, e incluso, que la de relictos de bosque seco (7).

La diversidad estructural y taxonómica de las especies vegetales permite soportar una alta diversidad de otros organismos, lo que hace importante como práctica el aumento de la riqueza de plantas en los diferentes subsistemas de producción. Es así, como los policultivos, potreros arborizados y potreros con sucesión vegetal, sostienen una biota variada a la vez que promueven un uso eficaz de los nutrientes del suelo. En el caso específico de los sistemas ganaderos, una mayor diversidad de plantas se promueve mediante la eliminación de quemas y herbicidas. El mantenimiento de los pastizales se efectúa a través de podas y corte selectivo de la vegetación (3).

En Colombia existen muchas especies arbóreas forrajeras distribuidas a lo largo de todos los pisos térmicos y que vienen siendo utilizadas por la ganadería como fuente de alimento y como elementos de conservación, bien sea plantadas en potreros como pequeños parches boscosos o en cercas vivas, las más comunes en los sistemas ganaderos de la región Caribe son: Leucaena leucocephala (leucaena), Gliricidia sepium (matarratón), Erythrina glauca (cantagallo), Piptadermia flora (guacamayo), Enterolobium cyclocarpum (orejero), Caesalpinia coriaria (dividivi), Guazuma ulmifolia (guácimo), Spondias mombin (jobo), Pithecellobium saman (campano) y Crescentia cujete (totumo) (8).
Crescentia cujete posee gran aceptación por diferentes especies animales conocida también como cujete, jícaro, mate, cutuco, calabazo, calabacero o huacal (9); originario de México y está disperso desde la Florida en Estados Unidos hasta Brasil y se extiende por Europa y Asia Tropical (10). Es un árbol de hasta de $10 \mathrm{~m}$ de altura, $30 \mathrm{~cm}$ de DAP (diámetro a la altura del pecho), las ramas usualmente torcidas, copa abierta, ramitas por lo general ausentes, con ramas muy extendidas y corteza corchosa fisurada $(11,12)$. El fruto es leñoso, variable en forma: redondos y alargados, y tamaño variado, por lo general entre 20 y $50 \mathrm{~cm}$ de diámetro; contiene pulpa blanca que encierra numerosas semillas elipsoides delgadas sin alas de 7- $8 \mathrm{~mm}$ de largo y 4-6 $\mathrm{mm}$ de ancho, esparcidas a través de una pulpa blanca, gelatinosa y esponjosa (13). Se estiman producciones de 16.2 a $81.2 \mathrm{~kg}$ de fruto por árbol; en Colombia se recolectan frutos a lo largo de todo el año (14).

En el presente estudio se evalúo la biodiversidad faunística asociada a ganadería vacuna de doble propósito en dos potreros estructurados con Botriochloa pertusa (colosoana) como herbácea dominante, uno de ellos plantado con Crescentia cujete (Bignoniaceae) como fuente de alimento adicional y otro con escaso y disperso arbolado, con ausencia de $C$. cujete, y se discute la importancia de incluir especies arbóreas nativas en áreas de pastoreo como alternativa de manejo sostenible y conservación de la diversidad de fauna silvestre local.

\section{MATERIALES Y MÉTODOS}

Sitio de estudio. El área de trabajo estuvo ubicada en la hacienda La Florida, área rural del municipio de Pinto, Magdalena, Colombia, tipificada como trópico bajo, formación vegetal bosque seco tropical (bsT) zona agrofísica subhúmeda con relieve de colinas y bien drenadas $(\mathrm{Cv}, \mathrm{Cu})$, clima cálido, temperatura promedio anual $28^{\circ} \mathrm{C}$, precipitación media $1.000-1.200 \mathrm{~mm}$, humedad relativa $75 \%$, suelos franco arcillosos (15). 
Evaluación de la fauna. La evaluación de la fauna silvestre presente se condujo en dos potreros, denominados: N1, que representó un sistema agrosilvopastoril compuesto por 500 árboles de $C$. cujete, de 10 años de edad; y N2, con 20 árboles adultos, que incluyeron: siete unidades de Tabebuia rosea, siete unidades de Gliricidia sepium y seis unidades de Pithecellobium saman. Los dos potreros, de 10 ha cada uno, presentaron como gramínea predominante Botriochloa pertusa (kikuyina o colosoana). En cada potrero se mantuvieron 15 vacas paridas - preñadas en el último tercio de la lactancia, doble propósito ( $1 / 2$ Cebú, $1 / 4$ Pardo y $1 / 4$ Costeño con Cuernos). La separación entre las dos áreas de muestreo fue de $500 \mathrm{~m}$.

Recolección de los datos. Mediante el método de punto fijo de conteo (16), durante 20 días continuos, del 1 al 20 de junio de 2008, mediante la aplicación de 10 sitios de avistamiento para cada área, escogidos al azar y con cubrimiento visualmente individual de $5.000 \mathrm{~m}^{2}$, se llevaron cabo las observaciones de avifauna y mastofauna. Los registros de herpetofauna se hicieron mediante colecta manual (17), usando la misma área referida, y se tuvo en cuenta las observaciones desde los puntos fijos de conteo cuando la detección de especímenes fue posible de este modo.

Los periodos de observación se condujeron entre las 06:00 y las 12:00 hr, las colectas manuales se realizaron entre las $14: 00$ y las 16:00 hr, se trabajó con dos observadores en cada punto fijo de conteo y se utilizaron binoculares Pentax $\AA 20 \times 60$ PCF WP II, bastones y pinzas de colecta.

Para el establecimiento del orden jerárquico en los diferentes listados de fauna tetrápoda se siguió la propuesta para anfibios de Darrel Frost del American Museum of Natural History-AMNH (vers. 5.2, julio 15 de 2008), para reptiles la propuesta en The Reptile Database de Peter Uetz (octubre 15 de 2008). Para aves la propuesta del South American Classification Comité, American Ornithologist'Union, (vers. 11, diciembre 2008). Para mamíferos los nombres comunes utilizados fueron obtenidos regionalmente y con apoyo en los registros para Familias de Polo-Urrea (18).

Análisis estadístico. Para determinar la existencia de similaridad entre las comunidades registradas en las dos áreas de trabajo se aplicó el índice de similitud de Sorensen (ISS) (19); para determinar un indicador de biodiversidad entre ambas comunidades se recurrió al índice de diversidad de Berger-Parker (IDBP) (1/d) (20), y para establecer diferencia significativa se aplicó una prueba de $\mathrm{X}^{2}$ teniendo en cuenta el número de especies por taxones y el número total de individuos de la comunidad. Al ganado vacuno adulto presente en las dos áreas de trabajo se les realizó un pesaje individual al inicio del trabajo y otro al final del mismo; los pesajes se realizaron con una báscula electrónica TRU-TEST ${ }^{\circledR}$ con capacidad de $1500 \mathrm{~kg}$ ( \pm 0.5 ). Se aplicó la prueba t de Student para comparar los pesos (21).

\section{RESULTADOS}

La identificación y registro numérico de la fauna silvestre hallada en el presente trabajo se detalla en las tablas 1,2 y 3 . Al aplicar el índice de similitud de Sorensen (ISS)(19) se obtuvo como valor para la herpetofauna $0.70,0.74$ para avifauna y 0.60 para mastofauna; la comunidad en total presentó un ISS equivalente a 0.71. Esto significa que ambas comunidades se parecen cualitativamente, ya que la mayor parte de las especies aparece en ambas. En cuanto al índice de diversidad de Berger-Parker (20) tomando la totalidad de la comunidad evaluada para las dos áreas de estudio se obtuvo: $\mathrm{N} 1=$ $0.083(1 / \mathrm{d}=12.04)$ y $\mathrm{N} 2=0.147(1 / \mathrm{d}$ $=6.80)$.

Porcentualmente, entre N1y N2 hubo una diferencia del $55 \%$ en cuanto al número de individuos de fauna silvestre registrados y del $57.7 \%$ con referencia al número de especies, siendo mayor la diversidad especifica y numérica en N1, como se puede observar mediante la prueba estadística para número de individuos $(p<0.05)$ y para especies 
Tabla 1. Avifauna registrada en las dos áreas de muestreo.

\begin{tabular}{|c|c|c|c|c|c|c|}
\hline Taxón & Orden & Familia & Especie & N. común & N (1) & $N(2)$ \\
\hline \multirow[t]{39}{*}{ AVES } & PELECANIFORMES & Anhingidae & Anhinga anhinga & pato aguja & 5 & 2 \\
\hline & CICONIFORMES & Ardeidae & Ardea cocoi & garza azul & 1 & 0 \\
\hline & & & Casmerodius albus & garza real & 1 & 1 \\
\hline & & & Egretta thula & garza patiamarilla & 2 & 1 \\
\hline & & & Bubulcus ibis & garcita ganadera & 33 & 34 \\
\hline & & & Tigrisoma lineatum & vaco & 1 & 0 \\
\hline & CATHARTIFORMES & Cathartidae & Coragyps atratus & golero & 8 & 7 \\
\hline & & & Cathartes aura & laura & 5 & 6 \\
\hline & ANSERIFORMES & Anatidae & Dendrocygna viduata & viudita & 3 & 2 \\
\hline & & & Dendrocygna autumnaliis & pisingo & 1 & 1 \\
\hline & GALLIFORMES & Odontophoridae & Colinus cristatus & perdiz & 23 & 14 \\
\hline & FALCONIFORMES & Accipitridae & Bursarellus nigricollis & águila cienaguera & 1 & 0 \\
\hline & & & Buteo nitidus & águila barrada & 1 & 0 \\
\hline & & Falconidae & Caracara plancus & caracara & 3 & 2 \\
\hline & & & Milvago chimachima & pigua & 5 & 6 \\
\hline & & & Herpetotheres cachinnans & halcón culebrero & 1 & 0 \\
\hline & & & Falco peregrinus & halcón peregrino & 1 & 1 \\
\hline & COLUMBIFORMES & Columbidae & Patagioenas cayennensis & guarumera & 3 & 1 \\
\hline & & & Columbina minuta & tortolita & 9 & 4 \\
\hline & & & Columbina talpacoti & tórtola & 5 & 6 \\
\hline & PSITTACIFORMES & Psittacidae & Aratinga pertinax & cotorra & 16 & 11 \\
\hline & & & Brotogeris jugularis & perico & 22 & 24 \\
\hline & CUCULIFORMES & Cuculidae & Crotophaga ani & cocinera & 35 & 21 \\
\hline & APODIFORMES & Trochilidae & Amazilia saucerotti & chupaflor & 2 & 0 \\
\hline & & & Phaethornis anthophilus & carinegro & 1 & 0 \\
\hline & & & Anthracothorax nigricollis & pechinegro & 1 & 1 \\
\hline & & & Chlorostilbon gibsoni & esmeralda & 2 & 0 \\
\hline & & & Damophilla julie & damófila & 1 & 0 \\
\hline & PASSERIFORMES & Tyrannidae & Tyrannus melancholicus & chamaría & 8 & 4 \\
\hline & & & Pitangus sulphuratus & garrochero & 5 & 3 \\
\hline & & Troglodytidae & Troglodytes aedon & cucarachero & 2 & 0 \\
\hline & & & Campylorhynchus griseus & Chupa huevo & 11 & 6 \\
\hline & & Thraupidae & Thraupis episcopus & azulejo & 5 & 0 \\
\hline & & & Thraupis palmarum & papayero & 3 & 0 \\
\hline & & & Ramphocelus icteronotis & toche & 3 & 0 \\
\hline & & Emberizidae & Sporophila minuta & mochuelo & 2 & 0 \\
\hline & & & Sicalis flaveola & canario & 11 & 7 \\
\hline & & & Oryzoborus crassirostris & bajero & 1 & 0 \\
\hline & & Icteridae & Cacicus cela & oropéndola & 3 & 0 \\
\hline Total & 11 & 16 & 39 & & 246 & 165 \\
\hline
\end{tabular}

Tabla 2. Mastofauna registrada en las dos áreas de muestreo.

\begin{tabular}{|c|c|c|c|c|c|c|}
\hline Taxón & Orden & Familia & Especie & N. común & N (1) & N (2) \\
\hline \multirow[t]{7}{*}{ MAMÍFEROS } & DIDELPHIMORPHIA & Didelphidae & Didelphys marsupialis & chucha & 2 & 1 \\
\hline & CINGULATA & Dasypodidae & Dasypus novemcinctus & armadillo & 1 & 0 \\
\hline & VERMILINGUA & Myrmecophagidae & Tamandua mexicana & oso hormiguero & 1 & 0 \\
\hline & CARNIVORA & Canidae & Cerdocyon thous & zorra perro & 2 & 1 \\
\hline & RODENTIA & Sciuridae & Sciurus granatensis & ardilla & 5 & 0 \\
\hline & & Muridae & Mus musculus & ratón casero & 3 & 0 \\
\hline & LAGOMORPHA & Leporidae & Sylvilagus brasilensis & conejo & 6 & 2 \\
\hline Total & 6 & 7 & 7 & & 20 & 4 \\
\hline
\end{tabular}


Tabla 3. Herpetofauna registrada en las dos áreas de muestreo.

\begin{tabular}{|c|c|c|c|c|c|c|}
\hline Taxa & Orden & Familia & Especie & N. común & $\mathbf{N}(\mathbf{1})$ & N (2) \\
\hline \multirow[t]{9}{*}{ ANFIBIOS } & ANURA & Branchycephalidae & Eleutherodactylus bufoniformis & rana & 3 & 0 \\
\hline & & & Eleutherodactylus raniformis & rana & 2 & 1 \\
\hline & & Bufonidae & Rhinella marina & sapo & 12 & 3 \\
\hline & & & Rhinella granulosa & sapito & 5 & 1 \\
\hline & & Ceratophryidae & Ceratophrys calcarata & sapo cuerno & 1 & 0 \\
\hline & & Hylidae & Hypsiboas crepitans & rana & 11 & 6 \\
\hline & & & Hypsiboas pugnax & rana & 3 & 2 \\
\hline & & & Scinax ruber & rana & 9 & 3 \\
\hline & & Leptodactylidae & Leptodactylus bolivianus & rana saltona & 1 & 0 \\
\hline \multirow[t]{17}{*}{ REPTILES } & SQUAMATA & Iguanidae & Anolis auratus & lobito & 16 & 7 \\
\hline & & & Iguana iguana & iguana & 29 & 8 \\
\hline & SUBORDEN SAURIA & Corytophanidae & Basiliscus basiliscus & guataquí & 2 & 0 \\
\hline & & Scincidae & Mabouya mabuya & lisa & 1 & 0 \\
\hline & & Teiidae & Ameiva ameiva & lobo & 9 & 3 \\
\hline & & & Ameiva festiva & lobo & 15 & 9 \\
\hline & & & Cnemidophorus lemniscatus & lobito & 16 & 12 \\
\hline & & & Tupinambis teguixin & Lobo pollero & 6 & 4 \\
\hline & SUBORDEN OPHIDIA & Boidae & Boa constrictor & boa & 1 & 0 \\
\hline & & & Epicrates cenchria & víbora & 1 & 0 \\
\hline & & & Corallus ruschenbergerii & mataboga & 2 & 0 \\
\hline & & Colubridae & Liophis lineatus & guardacamino & 2 & 1 \\
\hline & & & Liophis melanotus & guardacamino & 0 & 1 \\
\hline & & & Oxybelis aeneus & bejuquillo & 2 & 1 \\
\hline & & & Pseudoboa neuwiedii & Culebra cabecita & 1 & 0 \\
\hline & TESTUDINES & Emydidae & Trachemys callirostris & hicotea & 3 & 0 \\
\hline & & Kinosternidae & Kinosternon scorpioides & tapaculo & 1 & 0 \\
\hline Total & 4 & 13 & 26 & & 154 & 62 \\
\hline
\end{tabular}

$(p<0.05)$ lo comprueba la existencia de diferencias significativas entre ambas áreas para los dos parámetros evaluados.

La media del peso de las vacas sometidas a pastoreos en las dos áreas de estudio fue de $403 \mathrm{~kg}$ (DS 5.71), no hubo diferencias significativas entre el peso de los dos lotes de ganado durante el tiempo de estudio $(p>0.05)$.

\section{DISCUSIÓN}

En general la investigación sobre fragmentación de hábitat naturales se ha direccionado a entender las consecuencias biogeográficas y cambios de hábitat acorde con el tamaño de la deforestación (22), pero los enfoques orientados al manejo práctico, reconstructivo o mitigativo de los recursos naturales renovables involucrados no han sido lo suficientemente planteados y aplicados (23), en especial cuando se hace referencia a las explotaciones ganaderas vacunas que siguen un modelo tradicional de tala, cultivo y establecimiento de pasturas, dentro de un sistema generalizado que practica la ganadería extensiva como patrón generalizado de trabajo y que no tiene en cuenta la diversidad biológica que impacta, como sucede en la zona de estudio $y$ en gran parte del territorio nacional.

Queda demostrado en este caso que la implementación en potreros arborizados con C. cujete son una alternativa de producción pecuaria sostenible que permite el manejo ganadero al tiempo que estructura refugios que ayudan a mantener significativamente la fauna silvestre 
regional, sin que se reduzca el peso de los animales sometidos a pastoreo, porque podría creerse erróneamente que habría una disminución del área disponible de pastoreo por la presencia de los arboles. Se concuerda con Murgueitio y Calle (3), quienes manifiestan que la diversidad estructural y taxonómica de especies vegetales soporta una alta diversidad de organismos, lo cual comparativamente es significativo en este trabajo.

Los resultados indican que entre un potrero estructurado con solo pasturas, como clásicamente se maneja este tipo de explotación pecuaria intensiva, y otro arborizado mínimamente no hay diferencias en cuanto a los rendimientos de pesos del ganado, pero si existen valores agregados que son importantes, entre ellos incremento de la diversidad biológica y mejor mantenimiento del ecosistema, lo que se relaciona de forma directa con la heterogeneidad espacial o disposición de microhábitat (3), y permite estructurar un proceso de conservación local sin ir en detrimento de la producción económica y social que tiene la zona, haciendo menos lesiva la explotación ganadera en términos ambientales.

La mayor diversidad de especies que se observó en el potrero arborizado con $C$. cujete, es una característica predecible de los sistemas silvopastoriles, allí se presenta una mayor oferta de alimento representada en las posibilidades de establecimiento de variados gremios tróficos, como frugívoros, nectatívoros, folívoros e insectívoros entre otros, lo cual no es factible de establecimiento en áreas cuya estructura característica sean pastizales $(7,24)$, como se demuestra en este estudio.

Por otra parte, los diferentes estratos que conforman el conjunto de árboles de $C$. cujete incrementan la productividad biótica del sistema, ya que desde el piso hasta la copa permiten que existan diferentes microhábitat que se asocian a distintas especies y poblaciones generando una mayor productividad del sistema total, con un incremento de la dinámica de movimiento de individuos de variadas especies, en especial para especies nativas con requerimientos de hábitat menos generalistas y que por lo tanto se reducen poblacionalmente o desaparecen con la implementación de pasturas exclusivas $(24,25)$.

Es aceptable que las leñosas al crecer en el mismo terreno interfieren el paso de la radiación solar al estrato herbáceo, con lo cual la producción de forraje podría verse afectada, razón por la cual la práctica ganadera tradicional los tala (26). No obstante, existen especies de pastos que son resistentes a este fenómeno que van desde sombra intensa a nivel medio de sombrío, como: Brachiaria decumbens, $B$. humidicola, $B$. brizantha (27) y en este caso Botriochloa pertusa, que bien pueden ser combinadas con potreros arborizados, en los cuales se mantenga un número de leñosas adecuadamente estructurado que permita un sombrío medio.

En cuanto a los refugios que ofrece el arbolado dentro de un potrero, se puede conceptuar que poseen cierto grado de conectividad dentro de una matriz agropecuaria fragmentada (28) que posee la capacidad de generar variados hábitat y nichos para la fauna silvestre (29) y por lo tanto, como estrategia son altamente satisfactorios para afrontar los desafíos actuales de los sistemas de producción animal, ya que conservan la base de recursos naturales bióticos y abióticos (30), que son la base para poder mantener de forma racional la ganadería moderna en el trópico.

En conclusión, se demuestra la importancia de este modelo pecuario como promotor de la conservación faunística local al convertirse en refugio de variadas especies silvestres, al tiempo que mantiene la productividad existente sin alterar el ambiente sustancialmente. 


\section{REFERENCIAS}

1. Grez AA, Zaviezo T, Reyes S. Shortterm effects of habitat fragmentation on the abundance and species richness of beetles in experimental alfalfa microlandscapes. Rev Chil Hist Nat 2004; 77(3):547-558.

2. Wright SJ, Zeballos $\mathrm{H}$, Domínguez I, Gallardo MM, C Moreno MC, Ibáñez R. Poachers Alter Mammal Abundance, Seed Dispersal, and Seed Predation in a Neotropical Forest. Conserv Biol 2000; 14(1):227-239.

3. Murgueitio E. Silvopastoral systems in the Neotropics. Proceedings of an International Congress on Silvopastoralism and Sustainable Management held in Lugo, España, 2004. (Accesado: junio 20 de 2010). URL disponible en: http:// bookshop.cabi.org/Uploads/Books/ PDF/9781845930011/9781845930011. pdf $\#$ page $=26$.

4. Arias F. Análisis econométrico del riesgo de extinción de las especies de fauna en Colombia: reptiles y peces dulceacuícolas. Lect Econ 2004; 61:7-34.

5. Etter A, McAlpine C, Wilson K, Phinn $\mathrm{S}$, Possingham $\mathrm{H}$. Regional patterns of agricultural land use and deforestation in Colombia. Agric Ecosyst Environ 2006b; 114:369-386.

6. Etter A, van Wyngaarden W. Patterns of Landscape Transformation in Colombia, with Emphasis in the Andean Region. Ambio 2000; 29:412-439.

7. Cárdenas G. Comparación de la composición y estructura de la avifauna en diferentes sistemas de producción. [Trabajo de grado] Cali, Valle: Facultad de Ciencias; Departamento de Biología, Universidad del Valle; 2000.
8. Murgueitio RE. Sistemas Agroforestales para la Producción Ganadera en Colombia. Fundación CIPAV. Cali 2008. (Accesado: enero 20 de 2010). URL disponible en: http://www.fao.org/ ag/AGA/AGAP/FRG/AFRIS/espanol/ Document/AGROF99/Murgueit.htm.

9. Zamora S, Garcia J, Bonilla G, Aguilar $H$, Harvey CA, Ibrahim M. Uso de frutos y follaje arbóreo en la alimentación de vacunos en la época seca en Boaco, Nicaragua. Agroforestería en las Américas 2001; 8:31-38.

10. Bridgewater $S$, Ibañez $A$, Ratter $J A$, Furley P. Vegetation classification and floristics of the savannas and associated wetlands of the Rio Bravo conservation and management area, Belize. Edinb J Bot 2002; 59(3):421-442.

11. Arango-Ulloa J, Bohórquez A, Duque CM, Maass LB. Diversity of the calabash tree (Crescentia cujete L.) in Colombia. Agroforest Syst 2009; 76: 543-553.

12. Bass J. Incidental agroforestry in Honduras: the jícaro tree (Crescentia spp.) and pasture land use. J Lat Am Geogr 2004; 3(1):67-80.

13. Botero ALM, Chamorro GC. Revisión general de los aspectos botánicos y productivos de Crescentia cujete (Linneo, 1753). [Trabajo de grado]. Sincelejo, Sucre: Universidad de Sucre, Facultad de Ciencias Agropecuarias, Departamento de Zootecnia; 2003.

14. Arango AJ. Uso de la diversidad del Totumo (Crescentia cujete L): un árbol multipropósito para Colombia y América Tropical. Memorias seminario 2003. (Acceso abril 4 de 2009). URL disponible en: http://www.reuna.unalmed.edu. co/temporales/memorias/especies/ SeminarioMedellin.htm. 
15. Corporación Colombiana de Investigación Agropecuaria. Atlas de los sistemas de producción bovina, módulo Región Caribe. Bogotá: Corporación Colombiana de Investigación Agropecuaria; 2002.

16. Horlent N, Juárez, MC, Arturi $M$. Asociación Argentina de Ecología Incidencia de la estructura del paisaje sobre la composición de especies de aves de los talares del noreste de la provincia de Buenos Aires. Ecol Austral 2003; 13:173-182.

17. Rabinowitz A. Manual de Capacitación para la Investigación de Campo y la Conservación de la Vida Silvestre. Bolivia: Editorial FAN; 2003.

18. Polo-Urrea CF. Guía de taxonomía y nombres comunes en español e inglés de las familias de tetrápodos en Colombia. Bistua 2006; 2(1):231-246.

19. Arakaki M, Cano A. Composición florística de la cuenca del río Ilo-Moquegua y Lomas de Ilo, Moquegua, Perú. Rev Peru Biol 2003; 10(1):5-19.

20. Abós FP. El pluricultivo y la presencia de márgenes mantienen la diversidad biológica en los agroecosistemas. Ecología 2002; 16:273-285.

21. Sandoval E, Morales G, Jiménez D, Pino LA, Urdaneta J, Araque C. Caracterización de las diferentes modalidades de producción del sistema de ganadería bovina de doble propósito del Municipio José Antonio Páez del estado Yaracuy, Venezuela. Rev Fac Agron 2007; 24:501-521.

22. Ries L., Fletcher RJ, Battin J, Sisk T D. Ecological responses to habitat edges: Mechanisms, Models, and Variability Explained. Annu Rev Ecol Evol Syst 2004; 35:491-522.
23. López-Barrera F. Estructura y función en bordes de bosques. Ecosistemas 2004; 13(1):67-77.

24. Lindenmayer DB, Fisher J, Cunninghan RB. Native vegetation cover thresholds associated with species responses. Biol Conserv 2005; 124:311-316.

25. Atauri JA, De Lucio JV. The role of landscape Structure in species richness distribution of birds, amphibians, reptiles and lepidopterans in Mediterranean landscapes. Landscape Ecol 2001; 16:147-159.

26. Hernández M, Guenni O. Producción de biomasa y calidad nutricional del estrato graminoide en un sistema silvopastoril dominado por samán (Samanea saman (Jacq) Merr). Zootecnia Trop 2008; 26(4):439-453.

27. Guenni O, Gil JL, Guédez Y. Growth, forage yield and light interception and use by stands of five Brachiaria species in a tropical environment. Trop Grasslands 2005; 39:42-53.

28. Chacón M, Harvey CA. Live fence and landscape connectivity in a neotropical agricultura landscape. Agroforest Syst 2006; 68:15-26.

29. Galindo-González J, Guevara S, Sosa VJ. Bat and bird generated seed rains at isolated trees in pastures in a tropical rainforest. Conserv Biol 2000; 14(6): 1693-1703.

30. Montagnini F, Ugalde L, Navarro C. Growth characteristics of some native tree species used in silvopastoral systems in the humid lowland of Costa Rica. Agroforest Syst 2003; 59:163-170. 